\title{
Physical Properties of Interaction Mediating Fields
}

\author{
Eliahu Comay \\ Charactell Ltd., Tel-Aviv, Israel \\ Email: elicomay@post.tau.ac.il
}

How to cite this paper: Comay, E. (2018) Physical Properties of Interaction Mediating Fields. Open Access Library Journal, 5: e4738.

https://doi.org/10.4236/oalib.1104738

Received: June 25, 2018

Accepted: July 16, 2018

Published: July 19, 2018

Copyright $\odot 2018$ by author and Open Access Library Inc.

This work is licensed under the Creative Commons Attribution International License (CC BY 4.0).

http://creativecommons.org/licenses/by/4.0/

\begin{abstract}
This work analyzes quantum fields that describe particles and quantum fields that mediate interaction between particles. Criteria for the acceptability of a quantum theory are explained and used. The main result states that no genuine particle mediates interaction between other particles. It is proved that Maxwellian radiation fields, namely photons, interact with electric charges but no genuine photon is involved in a bound state of atomic electrons or in the case where an electronic beam is scattered by an electrically charged target. The term virtual photons, which describes interaction mediating electromagnetic fields, indicates that the current literature implicitly agrees with this conclusion. Analogous results are obtained for the strong nuclear force, for the strong interactions and for the weak interactions.
\end{abstract}

\section{Subject Areas \\ Quantum Mechanics}

\section{Keywords}

Criteria for the Acceptability of a Quantum Theory, Quantum Particles, Quantum Fields, Interaction Mediating Fields

\section{Introduction}

Fields are a primary element of the present structure of theoretical physics. Classical physics discusses the role of fields in electromagnetic and in gravitational interactions [1]. It is recognized that the classical theory holds in cases where the distance between the interacting particles is quite long and that this theory is inadequate in important cases that belong to the microscopic world of elementary particles. Here quantum mechanics and quantum field theory (QFT) take place. Elementary particle theory examines three kinds of primary interactions 
called strong, electromagnetic and weak, respectively.

The electromagnetic fields and their Maxwell equations are analyzed in classical physics and in quantum physics as well. Here the quantum theory introduces the wave-particle duality which is demonstrated by the photon. Moreover, the realm of quantum theories contains two new kinds of extension of the concept of fields of classical physics:

- According to the de Broglie idea, an elementary massive particle satisfies a wave equation (see [2], p. 3). This idea boils down to a description of such a particle by means of a specific field which satisfies an appropriate quantum equation.

- Quantum theories also aim to describe two new fundamental kinds of force which are called strong interactions and weak interactions, respectively. These interactions are found in nuclear and particle processes, and they have no classical analogue. Each of these forces is described by means of a field that satisfies an appropriate quantum equation.

Classical electrodynamics can be derived from the variational principle. Here the motion of an electric charge is derived from a Lagrangian (see [1], pp. 49-51) and a Lagrangian density of the electromagnetic fields is used for a derivation of Maxwell equations of the electromagnetic fields (see [1], pp. 71-80 or [3], p. 596)

$$
\mathcal{L}_{E M}=-\frac{1}{16 \pi} F^{\mu v} F_{\mu v}-j^{\mu} A_{\mu},
$$

where $F^{\mu v}$ is the electromagnetic fields tensor, $A_{\mu}$ is their 4-potential and $j^{\mu}$ is the 4-current of the electric charge. The last term of (1) describes the interaction between electrically charged matter and electromagnetic fields.

Quantum theories take an analogous form. For example, the 4-current of an electrically charged Dirac particle is (see [4], pp. 23, 24)

$$
j^{\mu}=-e \bar{\psi} \gamma^{\mu} \psi,
$$

where $e$ denotes the electric charge and $\bar{\psi}=\psi^{\dagger} \gamma^{0}$. This 4-current is substituted into the last term of (1), which describes the interaction of electromagnetic fields and an electrically charged Dirac particle.

The present work analyzes the structure of the interaction term of a quantum particle with the field that represents a specific force. It aims to prove that appropriate fields mediate interaction between particles but no genuine particle mediates interaction.

Relativistic expressions are written in the standard notation and the diagonal metric is $(1,-1,-1,-1)$. Units where $\hbar=c=1$ are used. In this system of units there is just one dimension and the concept of length $[L]$ is used. Thus, the expression $\left[L^{n}\right]$, where $n$ is a real number, denotes the dimension of a given physical quantity. The Second Section explains constraints that apply to physical theories. These constraints are used later in this work. The Third Section discusses electromagnetic interactions. The Fourth Section discusses the strong nuclear force. The Fifth Section discusses strong interactions. The Sixth Section 
discusses weak interactions. The Seventh Section contains some general remarks and the last section summarizes the paper.

\section{Constraints That Are Derived from Physical Principles}

As stated above, the purpose of the present work is to examine the consistency of the physical idea stating that a physically valid particle mediates an interaction between other physical particles. This work applies several constraints that should be satisfied by specific physical theories whose consistency is analyzed. This section describes how these constraints are deduced from well-established physical principles.

1) Special relativity is a well-established physical theory. For example, modern accelerators that are designed in accordance with this theory work successfully, and their data provide an astronomical number of examples whose analysis agrees with this theory. Therefore, special relativity is a cornerstone of this work and appropriate expressions are written in a relativistically covariant form. In particular, if two particles have different relativistic properties then they are inherently different physical entities. (Newtonian mechanics and quantum mechanics are acceptable because these theories hold for the corresponding relativistic theories in cases where $p / m \ll 1)$.

2) The variational principle plays a central role in the following discussion. This principle is adopted by many modern textbooks on field theory [1] [5]. For example, it is stated that the variational principle and its Lagrangian function are "the foundation on which virtually all modern theories are predicated" (see [6], p. 353). For this reason, the Lagrangian density $\mathcal{L}$ is used below as the cornerstone of the discussion.

The action

$$
S=\int \mathcal{L} \mathrm{d}^{4} x
$$

has the dimensions of $\hbar$ and in the unit system used herein it is a dimensionless quantity. Evidently, $\mathrm{d}^{4} x$ is a Lorentz scalar. It follows that every term of the Lagrangian density $\mathcal{L}$ must be a Lorentz scalar whose dimension is $\left[L^{-4}\right]$.

Conclusion: the mathematical structure of the Lagrangian density determines the dimension of the quantum functions that are described by it.

3) Wigner's analysis of the irreducible representations of the inhomogeneous Lorentz group [5] [7] [8] [9] demonstrates important results of special relativity. His work proves that a massive quantum particle has well defined mass and spin. A massless particle belongs to a different category. By definition, it has a zero mass and instead of spin, it has two components of helicity. Furthermore, in the metric used herein, the square of the 4-momentum of a physical particle takes the following values $p^{\mu} p_{\mu} \geq 0$. It means that there is no physical particle whose 4-momentum yields $p^{\mu} p_{\mu}<0$ (such a 4 -vector is call space-like).

Corollary: two physical objects that have a different mass or a different spin 
do not represent the same particle.

4) Let $x$ denotes a single set of the four space-time coordinates $(t, \boldsymbol{x})$. A quantum function of the form $\psi(x)$ represents an elementary quantum particle. This form indicates that an elementary quantum particle is pointlike. Indeed, a description of a composite quantum particle needs four independent variables $x$ for a description of the probability of finding the particle at $x$ and other degrees of freedom for a description of the distribution of the composite particle at the vicinity of $x$. This matter is agreed by the general community and QFT textbooks use $\psi(x)$ for a description of an elementary quantum particle [5] [10].

It follows that a description of a composite particle by a function of the form $\psi(x)$ can be regarded as an acceptable approximation in cases where the typical distance between particles is much larger than the actual size of the composite particle. A phenomenological approach may also be used in cases where the internal structure of the composite particle changes only marginally in the interaction process. For example, nuclear physics is based on the latter point.

Corollary: Since the function of an interaction mediating particle takes the form $\phi(x)$, one concludes that such a particle must be an elementary pointlike particle.

5) The generalized correspondence principle is used below as an element of the discussion. This principle states that an appropriate limit of a given theory should agree with the laws of a lower rank theory. The limit procedure demonstrates that the correspondence principle has a solid mathematical foundation. The present work applies correspondence relations between four physical theories: QFT; Relativistic quantum Mechanics (RQM); Quantum Mechanics (QM); Non-relativistic Classical Physics (CP) (see Figure 1). Non-relativistic classical physics is the classical limit of QM. This relationship is called the Bohr correspondence principle. QM is the nonrelativistic limit of RQM. RQM is the limit of QFT which holds for a process where the number of particles can be regarded as a constant of the motion. This chain of relations means that appropriate limits of QFT should agree with RQM, QM and CP.

The Bohr correspondence principle, which is a specific example of this principle, is discussed in many quantum mechanics textbooks (see e.g. [2], p. 15; [11], pp. 27-29). The Schroedinger equation is the non-relativistic limit of the Dirac equation (see e.g. [2], p. 330). The correspondence between QFT and QM is pointed out in S. Weinberg's QFT textbook: "First, some good news: quantum field theory is based on the same quantum mechanics that was invented by Schroedinger, Heisenberg, Pauli, Born, and others in 1925-26, and has been used ever since in atomic, molecular, nuclear and condensed matter physics" (see [5], p. 49). Hereafter, this relationship is called "Weinberg correspondence principle”. Many examples of the generalized correspondence principle are presented on pp. 1-6 of [12].

6) Consider a massive classical particle in its rest frame. Its 4-velocity is 


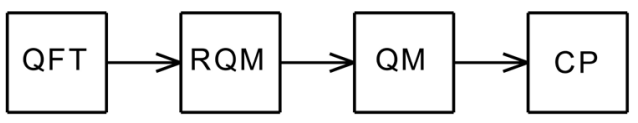

Figure 1. Correspondence between 4 theories. The arrows denote a limit transition between a higher theory and a corresponding lower theory (see text).

$$
v^{\mu}=(1,0,0,0),
$$

and the contraction of (4) with itself yields the following invariant (see [1], p. 23)

$$
v^{\mu} v_{\mu}=1 \text {. }
$$

The 4-derivative $a^{\mu}$ of $v^{\mu}$ with respect to the invariant time is the 4-acceleration. Relation (5) proves that

$$
v^{\mu} a_{\mu}=0
$$

(see [1], p. 24). It means that in Minkowski space the 4 -acceleration of a particle is orthogonal to its 4 -velocity.

By the Newton law, the 4-accelaration is related to the 4-force. Both the 4 -velocity and the 4-acceleration are 4-vectors. Hence, the 4-force must take the form of a second rank tensor $f^{\mu v}$

$$
m a^{\mu}=f^{\mu v} v_{v},
$$

where $m$ denotes the particle's self mass. If the 4 -force depends on an interaction mediating field then it is independent of physical properties of the particle on which the 4 -force is exerted.

A contraction of the 4-force (7) with the particle's 4-velocity and the orthogonality of (6) yield

$$
m a^{\mu} v_{\mu}=f^{\mu v} v_{v} v_{\mu}=0 .
$$

Since $f^{\mu v}$ is independent of $v_{\mu}$, one finds that the classical limit of $f^{\mu v}$ must be a second rank antisymmetric tensor. The dimension of the left-hand side of (7) and the unit system used herein prove that the dimension of $f^{\mu v}$ must be $\left[L^{-2}\right]$.

The continuity of the limit procedure which is an element of the correspondence principle of item 1 proves that it does not alter the tensorial structure and the dimension of the 4 -force of (8). Therefore, the mediating field of an interaction must be consistent with this 4 -tensor. The tensor of the electromagnetic fields satisfies these requirements (see [1], p. 65 or [3] p. 550).

7) The de Broglie principle states that a particle has wavelike properties where its wavelength is related to its momentum (see [2], p. 3)

$$
\lambda=2 \pi \hbar / p .
$$

It follows that a free quantum particle has a phase whose form is described by one of the following factors of its wave functions (see [2], p. 18)

$$
\cos (k x-\omega t), \sin (k x-\omega t), \mathrm{e}^{ \pm(k x-\omega t)} .
$$


8) The last principle of this list says that a physically acceptable theory should be consistent with experimental data that belong to its domain of validity. The solid basis of this principle is demonstrated by engineers that rely on it and construct many kinds of successfully operating devices.

These constraints are used below as criteria for the acceptability of physical theories.

\section{Electromagnetic Interactions}

The electromagnetic interaction term of the Lagrangian density is (see [10], p. 78; [13], p. 84)

$$
\mathcal{L}_{E M}=-e \bar{\psi} \gamma^{\mu} A_{\mu} \psi
$$

This expression describes the interaction of a charged Dirac particle with a photon as well as its interaction with the fields of another electrically charged particle. The 4-momentum of a real photon $p^{\mu}=(E, \boldsymbol{p})$ satisfies (see [4], p. 121; [6], p. 241)

$$
p^{\mu} p_{\mu}=E^{2}-p^{2}=0
$$

This relation means that the photon is a massless particle that travels at the speed of light in every inertial frame. Relation (12) is consistent with the experimental upper bound of the photon mass which is smaller by about 24 orders of magnitude of the electronic mass [14]!

A genuine photon is a particle that its creation is independent of the measurement process. For example, a photon that arrives from the sun was created more than 8 minutes before its measurement on earth and a photon that arrives from the Andromeda Galaxy was created more than one million years before it is measured on earth [15]. A photon measurement alters the state of a measuring device. For example, it can induce a transition of an atomic state (see [2] pp. 249-251), eject an electron in a Compton scattering (see [4], pp. 127-132), etc.

The photon is the quantum form of a Maxwellian radiation. Another kind of Maxwellian fields are the fields of interacting electric charges, like the fields of an atom where electrons are bound to the nucleus or the fields of an electron that is scattered on an electrically charged particle. Here the Maxwellian fields are bound to the interacting charges and act as a mediator of the interaction. The problem discussed in this section is whether the fields of a real photon represent the same particle as the fields that mediate interaction between electric charges.

One can find in the literature claims stating that these fields represent the same particle. For example: "But in quantum field theory, the electric field is quantized (in the form of photons), and we may picture the interaction as consisting of a stream of photons passing back and forth between the two charges, each electron continually emitting photons and continually absorbing them. And the same goes for any noncontact force: Where classically we interpret 'action at a distance' as 'mediated' by a field, we now say that it is mediated by an 
exchange of particles (the quanta of the field). In the case of electrodynamics, the mediator is the photon" (see [6], pp. 17, 18).

Several aspects of this problem are analyzed below. The analysis relies on the criteria that are described in Section 2.

1) Let us examine an electron scattered on an electrically charged target. The 4-momentum transfer $q^{\mu}$ is a useful concept of an analysis of such a process (see [16], pp. 189-191)

$$
q^{\mu}=p_{i}^{\mu}-p_{f}^{\mu},
$$

where $p_{i}^{\mu}, p_{f}^{\mu}$ denote the electron's initial and final 4-momentum, respectively. In the metric used herein, the square of the 4-momentum transfer satisfies

$$
q^{\mu} q_{\mu}=\left(E_{f}-E_{i}\right)^{2}-\left(\boldsymbol{p}_{f}-\boldsymbol{p}_{i}\right)^{2}<0 .
$$

Due to its negative value, such a 4-vector is called space-like.

Using Wigner's constraint 3 of Section 2, one finds that a comparison of the genuine photon of (12) and the four momentum transfer (14) proves that the four momentum transfer is not a genuine photon. It means that in a scattering process, photons do not mediate the interaction between electrically charged particles.

2) Let us examine the bound fields of any state of the hydrogen atom. Here the electronic state determines the angular momentum of the system (see [17], pp. 51-53). A better description also accounts for the hyperfine structure which evaluates the contribution of the proton state (see [17], pp. 107-114 or [18], p. 232). The consistency of the quantum description of atomic states means that bound electromagnetic fields do not contribute to the angular momentum of the system. The same is true for the parity of the atomic state. This outcome means that either of the following properties is true: Bound fields do not represent an independent quantum particle or the spin and the parity of such a particle are $j^{\pi}=0^{+}$.

By contrast the spin and the parity of the photon are $j^{\pi}=1^{-}$(see the photon data in [14]). Using Wigner's constraint 3 of Section 2, one finds that a genuine photon is not the same physical entity as bound fields.

3) Electromagnetic fields have two invariants (see [1], p. 68)

$$
I_{1}=B^{2}-E^{2}
$$

and

$$
I_{2}=\boldsymbol{E} \cdot \boldsymbol{B}
$$

Radiation fields represent photons. The invariant (15) vanishes for radiation fields emitted from a single source (see [1], pp. 119, 120 or [3], p. 657). The invariant (15) of the field of a single charge is easily found at the charge's rest frame. Here $\boldsymbol{E} \neq 0, \boldsymbol{B}=0$. Hence, the invariant $I_{1}<0$. This is yet another inherent difference between a real photon and bound fields.

The foregoing discussion shows several independent reasons where each of which explains why a real photon and bound electromagnetic fields do not 
represent the same physical entity. It means that photons exist. They interact with electric charges. On the other hand, if two charges belong to the system then the fields that mediate their electromagnetic interaction do not represent genuine photons. As a matter of fact, the literature contains expressions that implicitly agree with this conclusion. Thus, bound electromagnetic fields are called virtual photons (see [6], p. 63; [10], p. 5; [19], pp. 117-119). An analogous parlance says that virtual particles are not on their mass shell, namely, such a photon violates the relativistic energy-momentum relation of a genuine photon (12) (see [6], p. 213; [19], p. 119).

\section{The Strong Nuclear Force}

The Yukawa interaction aims to describe the strong nuclear force between two nucleons by means of a mediating Yukawa meson (see [13], p. 2; [20], p. 78). Physical properties of the Yukawa meson are derived from the Klein-Gordon (KG) Lagrangian density (see [13], p. 38)

$$
\mathcal{L}_{\mathrm{KG}}=g^{\mu v} \phi_{, \mu}^{*} \phi_{, v}-m^{2} \phi^{*} \phi .
$$

The nucleon-meson interaction term of the corresponding Lagrangian density takes the form (see [10], p. 116)

$$
\mathcal{L}_{\text {Yukawa }}=g \bar{\psi} \psi \phi,
$$

where $\bar{\psi}, \psi$ denote a nucleon and $\phi$ denotes a pion. This term is analogous to the electromagnetic interaction term (11). The literature refers to pions as the required Yukawa particles (see e.g. [4], p. 212; [20], p. 78). Each of the following arguments proves that the idea that a pion mediates nuclear interaction leads to a contradiction.

1) Experimental evidence proves that the strong nuclear force conserves parity (see [20], p. 4). On the other hand, the spin-parity of the pion are $j^{\pi}=0^{-}$(see the pion data of [14] or [16], p. 75). It follows that the Yukawa interaction term (18) violates parity. For this reason, the Yukawa interaction cannot describe the strong nuclear force.

2) Let us examine the classical limit of the Yukawa interaction. Constraint 1 of Section 2 proves that in this limit, the corresponding force of the Yukawa interaction must take the form of a second rank antisymmetric tensor whose dimension is $\left[L^{-2}\right]$. The pion is a Lorentz pseudoscalar and its Lagrangian density (17) proves that the dimension of its function $\phi$ is $\left[L^{-1}\right]$. It follows that the required antisymmetric tensor cannot be constructed on the basis of the pion's function $\phi$.

Therefore, the idea that a pion mediates nuclear interaction is inconsistent with the Weinberg and the Bohr correspondence principles of item 1 of Section 2.

3) Pions are not elementary particles. It is already well known that each of the three pions is a bound state of a quark and an antiquark of the $u, d$ flavor (see [6], pp. 176-178; [16], pp. 153-155). Furthermore, the pion is not a pointlike particle 
and its spatial size is not much smaller than that of the proton (see the $\pi^{ \pm}$data in [14] and apply isospin for the $\pi^{0}$ ).

Therefore, the idea that a pion mediates nuclear interaction is inconsistent with the requirement of item 4 of Section 2.

4) The following formula describes the radial dependence of the Yukawa potential (see [4], p. 211; [10], p. 122)

$$
V(r)=-\frac{g^{2}}{4 \pi} \frac{\mathrm{e}^{-m r}}{r},
$$

where $m$ denotes the pion mass and $g^{2}$ denotes the interaction strength. The distance dependence of the Yukawa potential is inconsistent with that of the experimentally measured nuclear potential. Indeed, the Yukawa potential (19) is attractive at every point and its intensity increases with the decrease if the distance between nuclei. At a short distance, the increase of the attraction is similar to that of the Coulomb potential. By contrast, the nuclear potential changes sign and becomes extremely repulsive at a short distance between nuclei (see [20], p. 97).

Therefore, the theoretical formula of the Yukawa interaction is inconsistent with the data.

5) The expression for density of a charged KG particle is

$$
\rho_{\mathrm{KG}}=i\left(\frac{\partial \phi}{\partial t} \phi^{*}-\phi \frac{\partial \phi^{*}}{\partial t}\right)
$$

(see [10], p. 18; [13], p. 40). This expression for density depends on derivatives of the quantum function $\phi$. On the other hand, the conserved expression of density of a non-relativistic Schroedinger particle is independent of derivatives

$$
\rho_{S c h}=\psi^{*} \psi
$$

(see [18], pp. 53-55). It means that the KG theory of a charged particle is inconsistent with the Weinberg correspondence principle of item 1 of Section 2. Furthermore, there is no expression for density of a neutral KG particle (see [21], pp. 41-43). This is yet another KG inconsistency with the Weinberg correspondence principle of item 1 of Section 2.

Conclusion: Each of the foregoing independent arguments proves that there is no theoretically valid support for the claim that pions mediate the nuclear force. Indications about the lack of a good theoretical basis for the Yukawa theory of the nuclear force can also be found in several textbooks. For example, one textbook refers to the Yukawa idea and says: "If you find that argument compelling, I can only say that you're pretty gullible" (see [6], problem 1.2 on p. 56). Authors of another textbook point out problems of the KG equation and state that "they appear with a vengeance" (see [13], p. 87).

\section{Strong Interactions}

Gluons are elements of the strong interaction theory called quantum chromodynamics (QCD), which are analogous to bound fields of the electromagnetic 
theory. On the other hand, there is no experimentally confirmed gluon state that is related to electromagnetic radiation fields (real photons), which are directly measured at places that are very far from the radiating system. Therefore, the validity of gluon existence depends on the consistency of QCD, which is the theory that requires this physical entity. The following items compare QCD with some well-known data and indicate its inconsistencies.

1) QCD has been constructed on an interpretation of baryons that contain three quarks of the same flavor, like the three uuu quarks of the $\Delta^{++}$baryon, whose spin-parity are $j^{\pi}=3 / 2^{+}$[14]. The primary QCD assertion says that each of these quarks is in a ground state $s$-wave. Hence, textbooks claim that these data demonstrate a fiasco of the Fermi-Dirac statistics of ordinary quantum mechanics (see [22], p. 5), because the three uuu quarks are fermions and their wave function must be antisymmetric. An analogous argument has also been used by one of the QCD pioneers, who refers to the three sss quarks of the $\Omega^{-}$baryon [23]. The following lines prove that contrary to the forgoing QCD assertion, the space-spin state of the $\Delta^{++}$baryon and of the $\Omega^{-}$baryon is not a symmetric single particle $s$-wave.

Figure 2 depicts the isospin doublet of the nucleons and the isospin quartet of the $\Delta$ baryons, and the valence quarks that compose each particle. Evidently, the same valence quarks compose the neutron and the $\Delta^{0}$. For this reason, the $\Delta^{0}$ is an excited state of the neutron. An analogous argument proves that the $\Delta^{+}$is an excited state of the proton. The excited state of the $\Delta^{0}, \Delta^{+}$baryons proves that the space-spin part of their wave function is not a symmetric ground state $s$-wave. Furthermore, all members of an isospin multiplet have the same space-spin structure (see [20], p. 73; [24], pp. 183-191). This claim relies on the fact that space-time coordinates are not affected by an isospin rotation. It follows that like the case of the $\Delta^{0}, \Delta^{+}$baryons, also the $d d d$ quarks of the $\Delta^{-}$baryon and the uuu quarks of the $\Delta^{++}$baryon are not in a symmetric ground state $s$-wave. An analogous argument holds for the $\Omega^{-}$baryon, where the 8,10 representations of the $\mathrm{SU}(3)$ group of the $u, d, s$ quarks respectively replace the isospin doublet and quartet representations of the SU(2) group of the $u, d$ quarks, which are depicted on Figure 2.

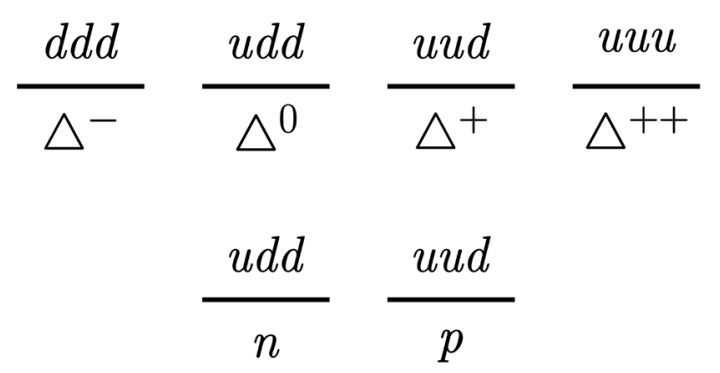

Figure 2. Baryonic isospin states and their energy levels: the $\mathrm{n}, \mathrm{p}$ nucleon doublet $(939 \mathrm{MeV})$ and the $\Delta$ quartet $(1232 \mathrm{MeV})$. The valence quarks that compose each particle are shown above its line (see text). 
These arguments prove that QCD has been constructed on the basis of an incorrect assertion. Evidently, it is very unlikely that such a theory can successfully describe physical data. The following points show some examples that illustrate this claim.

2) The data prove that the interaction of an energetic photon with a proton is about the same as its interaction with a neutron [25] [26]. Furthermore, the electromagnetic Compton interaction of a photon with the electric charge of the target makes a negligible contribution to the total cross section [26].

The photon is an important elementary particle and the nucleons are the best known hadronic states. However, QFT textbooks do not provide a theoretical explanation for the photon-nucleon interaction.

3) Nucleon measurements prove that quark density decreases exponentially with the increase of the radial distance $r$ (see [16], pp. 194-197). This is analogous to the electronic data of the hydrogen atom, where the Coulomb potential $V=-e^{2} / r$ determines the state (see [2], p. 85; [4], p. 55). By contrast, this radial decrease of quark density is inconsistent with QCD's asymptotic freedom, which claims that the attractive force increases with the increase of $r$ (see [6], p. 68 ; [10], pp. 425, 426).

4) The data prove that in a very high energy electron-proton scattering, the relative portion of elastic events is negligible and that the total cross section decreases as well (see [16], pp. 185-200, 262-275). It means that in nearly every collision, a quark that is struck violently by an electron makes an inelastic event. Now, QCD argues that quarks (together with quark-antiquark pairs) are the only massive constituents of a proton. Unlike electron-proton scattering, elastic events are found in many energetic proton-proton collisions and the total cross section as well as its elastic portion begins to increase with energy (see the proton-proton data on p. 11 of the 2012 PDG report [27]). Therefore, one wonders that if QCD is correct then what is the proton's component that takes the heavy blow of a high energy proton-proton collision and leaves the two protons intact? Why this proton component is not found in the corresponding electron-proton scattering? Why the elastic and the total cross sections of proton-proton scattering begin to increase with energy?

5) The following articles prove that QCD has many other inconsistencies [28] [29]. Moreover, a book that belongs to the popular science category explains many QCD contradictions in an easily understandable language [30].

In conclusion, the gluon is not a directly measured particle. Therefore, gluon existence depends on QCD's validity. The QCD inconsistencies suggest that gluons do not mediate strong interactions.

\section{The Electroweak Theory}

The electroweak theory aims to explain weak interactions [10] [31]. This theory argues that the three experimentally measured particles called $W^{ \pm}, Z$ mediate the weak interaction. For this reason, the validity of this claim stands on the 
self-consistency of the electroweak theory. The following points prove inconsistencies of this theory.

1) The $Z$ particle is a massive analogue of the photon (see [10], p. 701; [31], p. 307). Like the photon, this particle is electrically neutral and its quantum function takes a mathematically real form.

In quantum mechanics, the general form of the phase-dependent factor of a wave function uses the functions of (10). Hence, only the first and the second functions of (10), $\cos (k x-\omega t), \sin (k x-\omega t)$, contribute to a mathematically real quantum function. An application of simple trigonometry proves that the general form of the required mathematically real quantum function is

$$
\psi(t, x)=A \sin (k x-\omega t-\delta),
$$

where $A, \delta$ are mathematically real quantities which are a normalization factor and a phase displacement term, respectively.

Evidently, a free massive particle has a rest frame where its momentum $k=0$. In this frame the quantum function is

$$
\psi(t, x)=-A \sin (\omega t+\delta) .
$$

This result means that for every integer $n$, there is an instant $t$ when $\omega t+\delta=n \pi$, and the quantum function vanishes throughout the entire three-dimensional space. It follows that density cannot be defined for the $Z$ particle of the electroweak theory. It means that the theory of the $Z$ particle is inconsistent with the Weinberg correspondence principle of item 1 of Section 2, because the Schroedinger theory has a consistent expression for a conserved density of the wave function (see [2], pp. 21-24; [32], pp. 53-55).

2) The electroweak theory claims that beside the $Z$ particle, there are two electrically charged particles $W^{ \pm}$that mediate weak interactions. The dimension of these particles is $\left[L^{-1}\right]$, which is the same dimension as that of the $Z$ particle. By contrast, as stated in the previous item, the Schroedinger equation proves that in QM the product $\psi^{*} \psi$ represents density (see [2], pp. 21-24; [32], pp. 53-55). Hence, the dimension of the Schroedinger function is $\left[L^{-3 / 2}\right]$. It follows that the electroweak interpretation of the $W^{ \pm}$violates the Weinberg correspondence principle.

3) Other inconsistencies of the electroweak theory are discussed in Section 2 of [33].

The foregoing arguments disprove the electroweak claim that the $W^{ \pm}, Z$ bosons mediate weak interaction.

\section{Discussion}

Sections 3-6 contain specific arguments where each of which shows that a physical particle does not mediate an interaction between other particles. More general aspects of this issue are pointed out herein.

Item 2 of Section 5 describes experimental properties of a hard photon which interacts strongly with hadrons. It turns out that this important experimental 
information is ignored by most QFT textbooks. However, the literature contains an attempt aiming to explain this effect by means of an idea called the hadronic structure of the photon. The terms vector meson dominance (VMD) and vector dominance models (VDM) refer to this idea (see [25] [26] [34], pp. 317-320). VMD claims that an energetic photon is not a pure electromagnetic particle but a combination of a pure electromagnetic particle and a hadron (namely, a $j^{\pi}=1^{-}$vector meson)

$$
|\gamma\rangle=a\left|\gamma_{0}\right\rangle+b|h\rangle
$$

where $\left|\gamma_{0}\right\rangle,|h\rangle$ denote a pure electromagnetic photon and a hadron, respectively, and $a, b$ are normalization coefficients. In the case of a soft photon, $b$ is negligible and it becomes significant for a hard photon (see [25], p. 271; [34], pp. 317-320).

Evidently, the right-hand side of (24) is inconsistent with Wigner's analysis of item 3 of Section 2. Indeed, the photon is a massless particle and its angular momentum is called helicity. Helicity has two degrees of freedom. By contrast, a meson is a massive particle and the spin of a vector meson has three degrees of freedom. The different number of the spin degrees of freedom of the two terms that stand on the right-hand side of (24) negates the validity of the VMD idea. This is probably the underlying reason for the omission of this subject from QFT textbooks.

Let us turn to the strong nuclear force. This force is not regarded as a fundamental force [35]. Indeed, it is recognized as a residual force related to the strong interaction between quarks. For example, the following textbook states that "it is generally accepted that the force between nucleons is a facet of the strong interaction between quarks" (see [20], p. 100). The relationship between these forces is analogous to the electromagnetic force between electrons and the van der Waals force between neutral molecules (see [20], pp. 100-102). This claim is supported by the success of the nuclear liquid drop model (see [20], p. 139), and by the amazing similarity between the graph of the nucleon-nucleon potential (see [20], p. 97) and that of the effective potential between neutral molecules (see [36], p. 15). For the latter issue, see also the Lennard-Jones potential [37].

Pions are not elementary particles but bound states of a quark and an antiquark of the $u, d$ flavor. Therefore, the above mentioned analogy between the molecular and the nuclear forces means that the theoretical idea where a pion mediates the nuclear force is analogous to the totally infeasible idea stating that the ground state of the positronium mediates the molecular force. This conclusion casts doubts on the scientific merits of articles that interpret the nuclear force as an effect which is mediated by mesons in general and by pions in particular [38] [39].

\section{Concluding Remarks}

This work distinguishes between quantum fields that describe particles and fields that mediate interaction between particles. The analysis examines four 
kinds of interactions which are: the electromagnetic interaction, the strong nuclear force, the strong interactions and the weak interactions. These interactions are discussed in Sections 3-6, respectively. It is proved that no genuine particle mediates an interaction between particles. It means that the fields that mediate interaction do not represent a physically genuine particle. This is the primary result of the present work.

The following lines summarize the relevant points of each of the four interactions that are discussed above. Electrodynamics is certainly the best known field theory. Therefore, its summary is longer.

Electrodynamics provides the basis for a substantial portion of modern technological applications. It means that a fundamental part of this theory should be regarded as a quite reliable element of physics. Referring to the subject of this work, electrodynamics recognizes that radiation fields are made of quantum particles called photons. The helicity of each photon is unity and the relation between its energy and momentum satisfies (12). Such a photon interacts with matter. By contrast, there is a different kind of electromagnetic fields. These fields take part in effects like the bound states of atomic electrons or in a process where an electron is scattered by an electrically charged particle. Here electromagnetic fields mediate interaction between electric charges but these fields do not represent a genuine photon. The literature recognizes the difference between radiation fields that arrive as real photons which have been emitted from a remote source, and the fields that mediate interaction between electric charges. The latter kind of fields is called virtual photons or photons that are not on their mass shell. This state of affairs means that in the case of electromagnetic interactions, the present scientific community implicitly agrees with the results of this work.

Three other kinds of interactions are analyzed above: the strong nuclear force, strong interactions and weak interactions. This work also proves that there is no solid support for the idea that these forces are mediated by genuine particles.

\section{References}

[1] Landau, L.D. and Lifshitz, E.M. (2005) The Classical Theory of Fields. Elsevier, Amsterdam.

[2] Schiff, L.I. (1955) Quantum Mechanics. McGraw-Hill, New York.

[3] Jackson, J.D. (1975) Classical Electrodynamics. John Wiley, New York.

[4] Bjorken, J.D. and Drell, S.D. (1964) Relativistic Quantum Mechanics. McGraw-Hill, New York.

[5] Weinberg, S. (1995) The Quantum Theory of Fields, Vol. I. Cambridge University Press, Cambridge. https://doi.org/10.1017/CBO9781139644167

[6] Griffiths, D. (2008) Introduction to Elementary Particles. 2nd Edition, Wiley-VCH, Weinheim.

[7] Wigner, E. (1939) On Unitary Representations of the Inhomogeneous Lorentz Group. Annals of Mathematics, 40, 149. https://doi.org/10.2307/1968551 
[8] Schweber, S.S. (1964) An Introduction to Relativistic Quantum Field Theory. Harper \& Row, New York, 44-53.

[9] Sternberg, S. (1994) Group Theory and Physics. Cambridge University Press, Cambridge, 143-150.

[10] Peskin, M.E. and Schroeder, D.V. (1995) An Introduction to Quantum Field Theory. Addison-Wesley, Reading Mass.

[11] Messiah, A. (1967) Quantum Mechanics, V. 1. North Holland, Amsterdam.

[12] Rohrlich, F. (2007) Classical Charged Particle. World Scientific, New Jersey. https://doi.org/10.1142/6220

[13] Bjorken, J.D. and Drell, S.D. (1965) Relativistic Quantum Fields. McGraw-Hill, New York.

[14] Patrignani, C., et al. (2016) Particle Data Group. Chinese Physics C, 40, 100001. http://pdg.lbl.gov/

[15] https://en.wikipedia.org/wiki/Andromeda_Galaxy

[16] Perkins, D.H. (1987) Introduction to High Energy Physics. Addison-Wesley, Menlo Park.

[17] Bethe, H.A. and Salpeter, E.E. (1957) Quantum Mechanics of One- and Two-Electron Atoms. Springer, Berlin.

[18] Landau, L.D. and Lifshitz, E.M. (1959) Quantum Mechanics. Pergamon, London.

[19] Thomson, M. (2013) Modern Particle Physics. University Press, Cambridge. https://doi.org/10.1017/CBO9781139525367

[20] Wong, S.S.M. (1998) Introductory Nuclear Physics. Wiley, New York. https://doi.org/10.1002/9783527617906

[21] Berestetskii, V.B., Lifshitz, E.M. and Pitaevskii, L.P. (2008) Quantum Electrodynamics. 2nd Edition, Elsevier, Oxford.

[22] Halzen, F. and Martin, A.D. (1984) Quarks and Leptons. Wiley, New York.

[23] Fritzsch, H. (2012) The History of QCD. CERN Courier, Sep. 27. http://cerncourier.com/cws/article/cern/50796

[24] de-Shalit, A. and Talmi, I. (1963) Nuclear Shell Theory. Academic Press, New York.

[25] Bauer, T.H., Spital, R.D., Yennie, D.R. and Pipkin, F.M. (1978) The Hadronic Properties of the Photon in High-Energy Interactions. Reviews of Modern Physics, 50, 261. https://doi.org/10.1103/RevModPhys.50.261

[26] Weise, W. (1974) Hadronic Aspects of Photon-Nucleus Interactions. Physics Reports, 13, 53 .

[27] http://pdg.lbl.gov/2012/reviews/rpp2012-rev-cross-section-plots.pdf

[28] Comay, E. (2012) The Regular Charge-Monopole Theory and Strong Interactions. Electronic Journal of Theoretical Physics, 9, 93-118. http://www.ejtp.com/articles/ejtpv9i26p93.pdf

[29] Comay, E. (2004) A Regular Theory of Magnetic Monopoles and Its Implications. In: Chubykalo, A., Onoochin, V., Espinoza, A. and Smirnov-Rueda, R., Eds., Has the Last Word Been Said on Classical Electrodynamics, Rinton Press, Paramus, 82-103.

[30] Comay, O. (2014) Science or Fiction? The Phony Side of Particle Physics. Samuel Wachtman's Sons, Monterey.

[31] Weinberg, S. (1995) The Quantum Theory of Fields. Vol. II, Cambridge University 
Press, Cambridge.

[32] Landau, L.D. and Lifshitz, E.M. (1959) Quantum Mechanics. Pergamon, London.

[33] Comay, E. (2016) A Theory of Weak Interaction Dynamics. Open Access Library Journal, 3, 1-10.

https://www.scirp.org/journal/PaperInformation.aspx?PaperID=72788

[34] Frauenfelder, H. and Henley, E.M. (1991) Subatomic Physics. Prentice Hall, Englewood Cliffs, 296-304.

[35] https://en.wikipedia.org/wiki/Fundamental_interaction

[36] Haken, H. and Wolf, H.C. (1995) Molecular Physics and Elements of Quantum Chemistry. Springer, Berlin. https://doi.org/10.1007/978-3-662-03075-2

[37] https://en.wikibooks.org/wiki/Molecular_Simulation/The_Lennard-Jones_Potential

[38] Ishii, N., Aoki, S. and Hatsuda, T. (2007) Nuclear Force from Lattice QCD. Physical Review Letters, 99, Article ID: 022001.

https://doi.org/10.1103/PhysRevLett.99.022001

[39] Wilczek, F. (2007) Particle Physics: Hard-Core Revelations. Nature, 445, 156-157. https://doi.org/10.1038/445156a 